\title{
Use of aspirin for primary prevention in patients with diabetes during the COVID-19 pandemic
}

\author{
Chia Siang Kow ${ }^{1,2} \cdot$ Dinesh Sangarran Ramachandram ${ }^{2} \cdot$ Syed Shahzad Hasan ${ }^{3,4}$
}

Received: 11 September 2021 / Accepted: 15 September 2021 / Published online: 22 September 2021

(c) The Author(s), under exclusive licence to Royal Academy of Medicine in Ireland 2021

We read with interest the recently published cohort study by Tan and colleagues [1] which examined the appropriateness of aspirin prescribing among 400 patients with type 2 diabetes attending the Irish ambulatory clinics. It was reported that $49.0 \%(n=196)$ of the cohort were prescribed aspirin, of whom $10.2 \%(n=20)$ were receiving it for primary prevention of cardiovascular disease despite with $<10 \%$ of 10 -year cardiovascular risk. The authors specifically discouraged aspirin prescription in patients with type 2 diabetes who have $<10 \%$ of 10-year cardiovascular risk. Indeed, the recommendation of the authors coincided with the recommendation in the 2019 European Society of Cardiology (ESC)'s guidelines on diabetes, pre-diabetes, and cardiovascular diseases, which also discouraged the prescription of aspirin in patients with diabetes at low-to-moderate cardiovascular risk [2].

Nevertheless, the pleiotropic effects of aspirin have been well recognized in the literature; aspirin has been reported to possess anti-inflammatory, analgesic, antipyretic, antithrombotic effects, as well as antiviral properties against RNA viruses [3]. Indeed, during the coronavirus disease 2019 (COVID-19) pandemic, aspirin has been proposed as a treatment for COVID-19 based on its antithrombotic properties [4]. Therefore, we believe the recommendations on the use of aspirin in patients with diabetes should be revised in the current context where COVID-19 is still a serious global health threat despite the availability of COVID-19 vaccines. As reported in a meta-analysis [5] of observational studies,

Chia Siang Kow

chiasiang_93@hotmail.com

1 School of Postgraduate Studies, International Medical University, Kuala Lumpur, Malaysia

2 School of Pharmacy, Monash University Malaysia, Bandar Sunway, Subang Jaya, Selangor, Malaysia

3 School of Applied Sciences, University of Huddersfield, Huddersfield, United Kingdom

4 School of Biomedical Sciences \& Pharmacy, University of Newcastle, Callaghan, Australia the pre-diagnosis use of aspirin in patients with COVID-19 is associated with a significantly reduced risk of fatal course of COVID-19, relative to non-use of aspirin (pooled odds ratio $=0.50,95 \%$ confidence interval $0.32-0.77$ and pooled hazard ratio $=0.50,95 \%$ confidence interval $0.36-0.69$ ). In addition, a recently published observational cohort study [6] (not included in the meta-analysis) also reported significantly lower in-hospital mortality (hazard ratio $=0.81,95 \%$ confidence interval 0.76-0.87), with pre-hospitalization use of antiplatelets ( $83.9 \%$ were aspirin users) compared with non-use of antiplatelet therapy.

While we acknowledged the negative findings in the RECOVERY trial [7] in which the use of aspirin was not associated with reduction in 28-day mortality in patients with COVID-19 (rate ratio $=0.96,95 \%$ confidence interval $0 \cdot 89-1 \cdot 04$ ), it should be noted that diabetic patients constituted only $22 \%$ of the trial participants. Increased platelet activation has been described previously in patients with diabetes (regardless of diabetes control), which may be due to hyperglycemia, low-degree inflammation, and increased oxidation [8]. In addition, thrombin generation in platelets appears to be enhanced in patients with diabetes [9]. Thus, the development of COVID-19 in patients with diabetes may enhance the pre-existing platelet dysfunction, since COVID19 itself, and many of its complications, have been associated with platelet activation, mainly due to uncontrolled overproduction of inflammatory cytokines [10].

Therefore, in contrary with the current recommendation [2] and the findings by the authors [1], we opined that during the COVID-19 pandemic, the use of aspirin should be encouraged in patients with diabetes, even for those who are at low-to-moderate cardiovascular risk, to reduce the risk of COVID-19-related complications (including death). Thus far, the beneficial effects of aspirin in patients with COVID-19 had been associated with its pre-diagnosis use $[5,6]$; the prescription of aspirin after the development of COVID-19 may not be adequate to counteract the enhanced platelet dysfunction as reported in the RECOVERY trial 
[7]. Nonetheless, future trials with aspirin in patients with COVID-19 should aim to recruit those with concurrent diabetes to better ascertain its effects in this population.

\section{Declarations}

Conflict of interest The authors declare no competing interests.

\section{References}

1. Tan SY, Cronin H, Byrne S et al (2021) Appropriateness of aspirin prescribing for primary and secondary prevention of cardiovascular disease in type 2 diabetes in different care settings [published online ahead of print, 2021 Jun 22]. Ir J Med Sci. https://doi.org/ 10.1007/s11845-021-02649-5

2. Cosentino F, Grant PJ, Aboyans V et al (2020) ESC Guidelines on diabetes, pre-diabetes, and cardiovascular diseases developed in collaboration with the EASD [published correction appears in Eur Heart J. 2020 Dec 1;41(45):4317]. Eur Heart 41(2):255-323

3. Hybiak J, Broniarek I, Kiryczyński G et al (2020) Aspirin and its pleiotropic application. Eur J Pharmacol 866:172762
4. Rizk JG, Lavie CJ, Gupta A (2021) Low-dose aspirin for early COVID-19: does the early bird catch the worm? Expert Opin Investig Drugs 30(8):785-788

5. Kow CS, Hasan SS (2021) Use of antiplatelet drugs and the risk of mortality in patients with COVID-19: a meta-analysis. J Thromb Thrombolysis 52(1):124-129

6. Chow JH, Yin Y, Yamane DP et al (2021) Association of prehospital antiplatelet therapy with survival in patients hospitalized with COVID-19: A Propensity Score-Matched Analysis [published online ahead of print, 2021 Aug 29]. J Thromb Haemost. https://doi.org/10.1111/jth.15517

7. RECOVERY Collaborative Group, Horby PW, Pessoa-Amorim G (2021) Aspirin in patients admitted to hospital with COVID-19 (RECOVERY): a randomised, controlled, open-label, platform trial. Preprint. medRxiv 2021.06.08.21258132

8. Davì G, Catalano I, Averna M et al (1990) Thromboxane biosynthesis and platelet function in type II diabetes mellitus. N Engl J Med 322(25):1769-1774

9. Kearney K, Tomlinson D, Smith K, Ajjan R (2017) Hypofibrinolysis in diabetes: a therapeutic target for the reduction of cardiovascular risk. Cardiovasc Diabetol 16(1):34

10. Yatim N, Boussier J, Chocron R et al (2021) Platelet activation in critically ill COVID-19 patients. Ann Intensive Care 11(1):113 